\title{
Investigation of structural, optical and morphological properties of InGaN/GaN structure
}

\author{
A. Kürşat Bilgili ${ }^{1}$ Ömer Akpınar ${ }^{2} \cdot$ M. Kemal Öztürk ${ }^{1,2} \cdot$ Ceren Başköse $^{2} \cdot$ Süleyman Özçelik $^{1,2} \cdot$ Ekmel Özbay $^{3}$
}

Received: 21 October 2018 / Accepted: 15 December 2018 / Published online: 19 December 2018

(c) Springer-Verlag GmbH Germany, part of Springer Nature 2018

\begin{abstract}
In this study, InGaN/GaN structure is investigated in the temperature range of $300-500{ }^{\circ} \mathrm{C}$ with steps of $50{ }^{\circ} \mathrm{C}$. InGaN/ $\mathrm{GaN}$ multi-quantum well structure is deposited on c-orientated sapphire wafer by metal organic chemical vapour deposition method. All the parameters except for temperature kept constant during growth period. InGaN/GaN structures with different In content are investigated by XRD technique. Their structural, optical and morphological characteristics are determined by high resolution X-ray diffraction, Fourier transform spectroscopy (FTIR), photo luminescence (PL), transmission and atomic force microscopy (AFM). According to FTIR and PL spectra's, it is noticed that band gap values coincide with blue region in the electromagnetic spectrum. As a result of transmission measurements it is seen that light is completely absorbed by the sample at approximately $390 \mathrm{~nm}$. Using XRD technique, dislocation densities and strain are calculated. Full width at half maximum of the XRD peak values gained from X-ray diffraction are used in an alternative method called Williamson-Hall (W-H). Using W-H method, lateral and vertical crystal lengths and tilt angles are determined. Surface roughness parameters are investigated by AFM. Different properties of $\mathrm{GaN}$ and $\mathrm{InGaN}$ layers are compared as dependent on increasing temperature. According to AFM images it is seen that these structures have high surface roughness and large crystal size. All the results yielded from the mentioned methods are in good agreement with the previous works done by different authors.
\end{abstract}

\section{Introduction}

A. Kürşat Bilgili

ahmet.kursat.bilgili@gazi.edu.tr

Ömer Akpınar

omerakpinar9@gmail.com

M. Kemal Öztürk

ozturkmodev@gmail.com

Ceren Başköse

cerenbaskose@gmail.com

Süleyman Özçelik

sozcelik61tr@gmail.com

Ekmel Özbay

ozbay@bilkent.edu.tr

1 Department of Physics, Gazi University, 06500 Ankara, Turkey

2 Photonics Research Center, Gazi University, 06500 Ankara, Turkey

3 Nanotechnology Research Center, Bilkent University, 06800 Ankara, Turkey
High technology, especially nano technology has great importance in some industrial areas such as, communication, defence industry, transportation, medicals and digital electronics. Devices used in these areas are getting smaller and their performance increase with nano technology. Among these devices, two of the most important ones are lightemitting diodes (LEDs) and solar cells.

III-V group semiconductors with nitrides has a special place in production of optoelectronic devices. For example $\mathrm{InN}$ and $\mathrm{GaN}$ has band gaps as $0.7 \mathrm{eV}$ and $3.4 \mathrm{eV}$, respectively [1]. This property makes the nitride-based semiconductors very important in designing optoelectronic devices. Besides nitride-based semiconductors can operate on hard conditions such as high temperature and pressure [2].

MQWs are used in III-group nitride LED and LASER structures. In literature there are many studies on InGaN/ GaN MQWs. These are used in green, blue and UV LEDs with high-internal quantum efficiency. They are also used in InGaN and AlGaN. In UV-emitters these structures are very popular. Diffraction in super lattice may be understood by looking at the diffraction in terms of optics. Band gap of 
$\mathrm{GaN}$ is $3.4 \mathrm{eV}$ and band gap of InGaN varies from 0.7 to $3.4 \mathrm{eV}$. The value of band gap for InGaN changes according to In content in it. When these two alloys are used together because of the differences in band gaps they form a potential step called quantum well. Also in structural terms, studies showed that increasing the In content in the grade makes it columnar. In content $(x)$ values may change in the range of $0.13-0.17$. If this content takes values in steps in InGaN so it is graded. As In content $(x)$ increase the c-lattice parameter makes less tilt with $a-b$ plane in hexagonal structures. Also all graded samples are strongly optically active so these samples can also be used as solar cells.

White light can not be gained using LEDs directly. But by covering the InGaN LED with phosphorus we can gain white light. One approach to gain white light is phosphorconverted white light. Here white light is generated by use of phosphors with a short wavelength LED. If the phosphor in LED is illuminated by blue light, it emits yellow light having a fairly broad spectral power distribution. Using the phosphor in the body of LED with a peak wavelength of $450-470 \mathrm{~nm}$ some of the blue light will change to yellow light. The remaining blue light will be superimposed with yellow light and this superposition will produce white light $[3,4]$. Brightness, long life, low energy need, and other good properties can be gained using InGaN LEDs [4]. Also InGaN solar cells are preferred, because they have hexagonal structure and they are resistent against high temperature, frequency and pressure, but when we deposited it on sapphire $\left(\mathrm{Al}_{2} \mathrm{O}_{3}\right)$ a great lattice mismatch like $\% 15$ comes out. This big ratio of lattice mismatch may cause dislocations, tilt and mosaic defects. This situation may cause the device performance to deteriorate. For instance, these dislocations may come out in the range of $10^{7}-10^{11} \mathrm{~cm}^{-2}$ and this may result with cracks in the layers [5-7]. To come over such disadvantages, before depositing InGaN layer on sapphire, an interlayer may be deposited on sapphire to reduce lattice mismatch. After this step, structural, optical and electrical properties of the device improve. In these structures, because of the big ratio of lattice mismatch, active layers of InGaN may contain threadening dislocations (TD) in the range of $10^{18}-10^{12} \mathrm{~cm}^{-2}[8,9]$. Solar cell devices formed by III-nitrides can work even in presence of threadening dislocation densities as high as $10^{11} \mathrm{~cm}^{-2}$. This situation is caused by carrier localization. Although there is a practical importance the origin of TDs in III-nitrides are not fully explained yet. TDs may be screw, mixed or edge type. It is noticed that TD line direction is often parallel to [0001] by different authors. It is generally mentioned that great lattice mismatch is responsible for generation of TDs. But there are also different explanations for generation of TDs. For example TDs are formed by coalescence boundaries of misoriented islands during film growth, these islands forms sub-structures that are tilted and twisted with respect to each other. Another approach for formation of TDs is nucleation layer model. All the evidence for this model is obtained from AFM studies for partially coalesced GaN films [6]. Although these dislocations are in a great percentage, InGaN solar cells are still more effective than other types of solar cells. Because III-V group nitride-based optoelectronic devices are less sensitive to defects than traditional semiconductors [9]. In this study InGaN/GaN structure is investigated in terms of morphology and optics. Also some crystal structure properties are determined using HR-XRD technique.

\section{Experimental}

TMGa, TMIn and ammonia are decomposed into $\mathrm{Ga}$, In and $\mathrm{N}$, respectively in a MOCVD reactor (Aixtron 200/4 HT-S) under low pressure and at low temperature GaN (LT-GaN) layers are formed. Using trimethylgallium (TMGa), trimethylaluminum (TMAl) and ammonia sources (transport of these gases was formed with $\mathrm{H}$ source at high temperature), the InGaN material was formed on the $\mathrm{Al}_{2} \mathrm{O}_{3}$ substrate. Three different sample groups were placed and they were named as Sample A, B, C (S.A, S.B, S.C). Before epitaxial growth operation, $\mathrm{Al}_{2} \mathrm{O}_{3}$ wafer is annealed at $1100{ }^{\circ} \mathrm{C}$ for $10 \mathrm{~min}$ in nitrogen atmosphere to remove the oxide layer on its surface. The GaN (LT-GaN) layer at low temperature was to prevent mismatch in the first step. All samples contain LT-GaN layers at different thicknesses (Fig. 1). Five $\mathrm{GaN}$ layers were then grown as undoped (ud) buffer layer under constant pressure (200 mbar) to reduce dislocations. All of these stages are common among the three samples. Sample A, 2.45 min LT GaN, five ud-GaN ( $1.9 \mu \mathrm{m}$ in total), $1.9 \mu \mathrm{m} n-\mathrm{GaN}, 200 \mathrm{~nm}$ InGaN, $25 \mathrm{~nm}$ graded $\mathrm{InGaN}, 50 \mathrm{~nm}$ p-InGaN and $20 \mathrm{~nm}$ thick p-InGaN layers were grown. Sample B, 2.45 min LT GaN, five ud-GaN (1.9 $\mu \mathrm{m}$ in total), $1.9 \mu \mathrm{m} n-G a N, 220 \mathrm{~nm}$ InGaN, $50 \mathrm{~nm}$ p-InGaN and $20 \mathrm{~nm}$ thick p-InGaN layers were grown. Sample C, $3.30 \mathrm{~min}$ LT GaN, five ud-GaN (2.1 $\mu \mathrm{m}$ in total), $2.04 \mu \mathrm{m} \mathrm{n}-\mathrm{GaN}$, $20 \mathrm{~nm}$ InGaN, $160 \mathrm{~nm}$ graded InGaN, $20 \mathrm{~nm} \mathrm{InGaN,} 50 \mathrm{~nm}$ $\mathrm{p}$-InGaN and $20 \mathrm{~nm}$ thick p-InGaN layers were grown. As mentioned in many other studies, active layers can not be grown directly over sapphire substrate. There must be a buffer layer between active layers and sapphire as in our study (LT GaN layer). Otherwise if active layers are grown over sapphire directly there may form cracks that cause malfunction of the device.

\subsection{XRD analysis}

The XRD analysis of the samples are performed using (high resolution X-ray diffraction) HR-XRD D-8 discovery device. Advantages over normal XRD: It has 4 crystal Ge monochromator with 022 orientation, and this 
Sample A

p-InGaN layer $20 \mathrm{~nm}$
p-InGaN layer $50 \mathrm{~nm}$
Graded InGaN layer $25 \mathrm{~nm}$
InGaN layer $200 \mathrm{~nm}$
$\mathrm{n}-\mathrm{GaN}$ layer $1.9 \mu \mathrm{m}$
$\mathrm{Ud}-\mathrm{GaN}$ layer $1.9 \mu \mathrm{m}$
$\mathrm{LT}-\mathrm{GaN}_{2} 2.45$ min
$6 \mathrm{H}-\mathrm{Al}_{2} \mathrm{O}_{3}$ substrate

\section{Sample B}

p-InGaN layer $20 \mathrm{~nm}$ p-InGaN layer $50 \mathrm{~nm}$ InGaN layer $220 \mathrm{~nm}$ $\mathrm{n}-\mathrm{GaN}$ layer $1.9 \mu \mathrm{m}$ $\mathrm{Ud}-\mathrm{GaN}$ layer $1.9 \mu \mathrm{m}$ LT-GaN $2.45 \mathrm{~min}$ $6 \mathrm{H}-\mathrm{Al}_{2} \mathrm{O}_{3}$ substrate
Sample C

p-InGaN layer $20 \mathrm{~nm}$
p-InGaN layer $50 \mathrm{~nm}$
InGaN layer $20 \mathrm{~nm}$
Graded InGaN layer $160 \mathrm{~nm}$
InGaN layer $20 \mathrm{~nm}$
$\mathrm{n}-\mathrm{GaN}$ layer $2.04 \mu \mathrm{m}$
$\mathrm{Ud}-\mathrm{GaN}$ layer $2.1 \mu \mathrm{m}$
$\mathrm{LT}-\mathrm{GaN}_{3} .30$ min
$6 \mathrm{H}-\mathrm{Al}_{2} \mathrm{O}_{3}$ substrate

Fig. 1 LT-GaN epilayers grown on $6 \mathrm{H}-\mathrm{Al}_{2} \mathrm{O}_{3}$ substrate for sample A, B and C

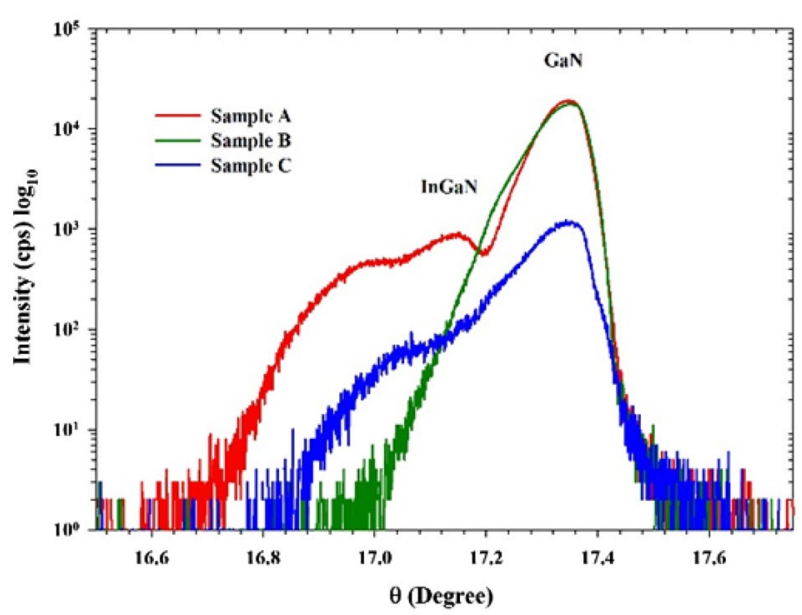

Fig. 2 HR-XRD $\omega$ curves of S.A, B and C in (0002) Miller reflection plane

monochromator uses high resolution peak with single wavelength by separating $K_{\alpha 1}$ and $K_{\alpha 2}$ function. $\mathrm{Cu} K_{\alpha 1}$ tube is used as a source. To obtain good diffraction condition in symmetric and asymmetric planes, $x-, y-, z-, \phi$ and $\chi$ axes are used other than $\theta$ and $2 \theta$ axes.

Figure 2 shows $\omega$ curves for three different samples. For S.A on the left of $\mathrm{GaN}$ top peak $\mathrm{InGaN}$ peak is not fully distorted. For S.B on the left of GaN peak InGaN peak is not clear. For S.C on the left of top GaN peak InGaN peak is approximately clear but as in S.A it is also not fully distorted. These undistorted InGaN peaks can be attributed to microstructural defects $[10,11]$. According to Vegard's law In content $(x)$ can be calculated by the following formula:

$x=\frac{c_{0}(\mathrm{InGaN})-c_{0}(\mathrm{GaN})}{c_{0}(\mathrm{InN})-c_{0}(\mathrm{GaN})}$

In contents for S.A are 0.105 and 0.183 , for S.B 0.136 and 0.249 and for S.C 0.048 and 0.207 in (first InGaN

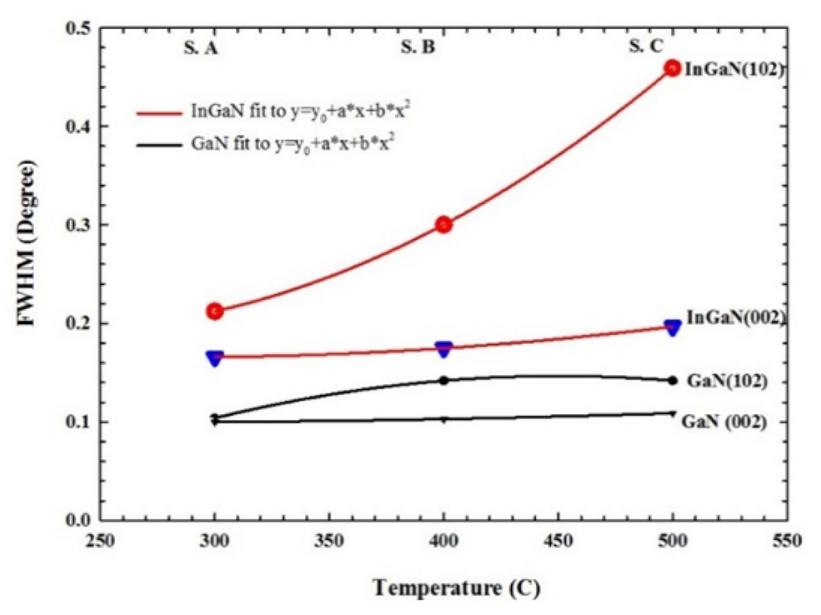

Fig. 3 Variation of FWHM dependent on temperature for $\mathrm{GaN}$ and InGaN layers in (002) symmetric and (102) asymmetric planes

layer) InGaN1 and (second InGaN layer) InGaN2 layers, respectively.

For samples A, B and C Fig. 3, for symmetric (002) plane and for (105) asymmetric planes, shows variation of FWHM dependent on temperature (T). For (002) symmetric plane, at increasing growth temperatures, $\mathrm{GaN}$ layer is at lower FWHM values than InGaN layer. FWHM value for GaN increase with the increase in temperature. FWHM value for InGaN layer increase more than for GaN layer. For (105) asymmetric plane, FWHM values first increase until $400{ }^{\circ} \mathrm{C}$ later its value slightly decrease with increasing temperature. For InGaN layer, in the same plane FWHM values only increase with increasing temperature. Values calculated for InGaN layer are higher than the ones for GaN layer. Increase in InGaN layer values are \%25 more than the increase in the values of GaN layer. Variation of FWHM may be caused from the difference in thermal expansion coefficients or lattice mismatch. The reason for choosing the $300-500{ }^{\circ} \mathrm{C}$ temperature range is that there were not any important variation in FWHM values until $300{ }^{\circ} \mathrm{C}$. FWHM values are related 
with particle size. Particle size may be detected different from normal values at different temperatures.

Also, Fig. 4 representing the whole scans for (002), (101), (102), (004), (121) and (006) planes, peak positions and FWHMs of InGaN and GaN layers can be seen. Using Fig. 4 FWHM values belonging to the same planes for three samples can be compared. In InGaN layer FWHM values are near to each other for S.B and C in (002) and (004) planes. FWHM value for S.A is clearly lower in both planes. In (102), (121) and (006) planes FWHM values are very near to each other for all three samples. For GaN layer, in (002) plane FWHM values for each sample is very different from each other as in-plane (101). For plane (102) and (004) FWHM values are similar to each other for all three samples. Variation in FWHM dependent on Bragg angles may be originated from defects such as dislocations in the samples or refraction of the X-rays probing into the samples with different angles.
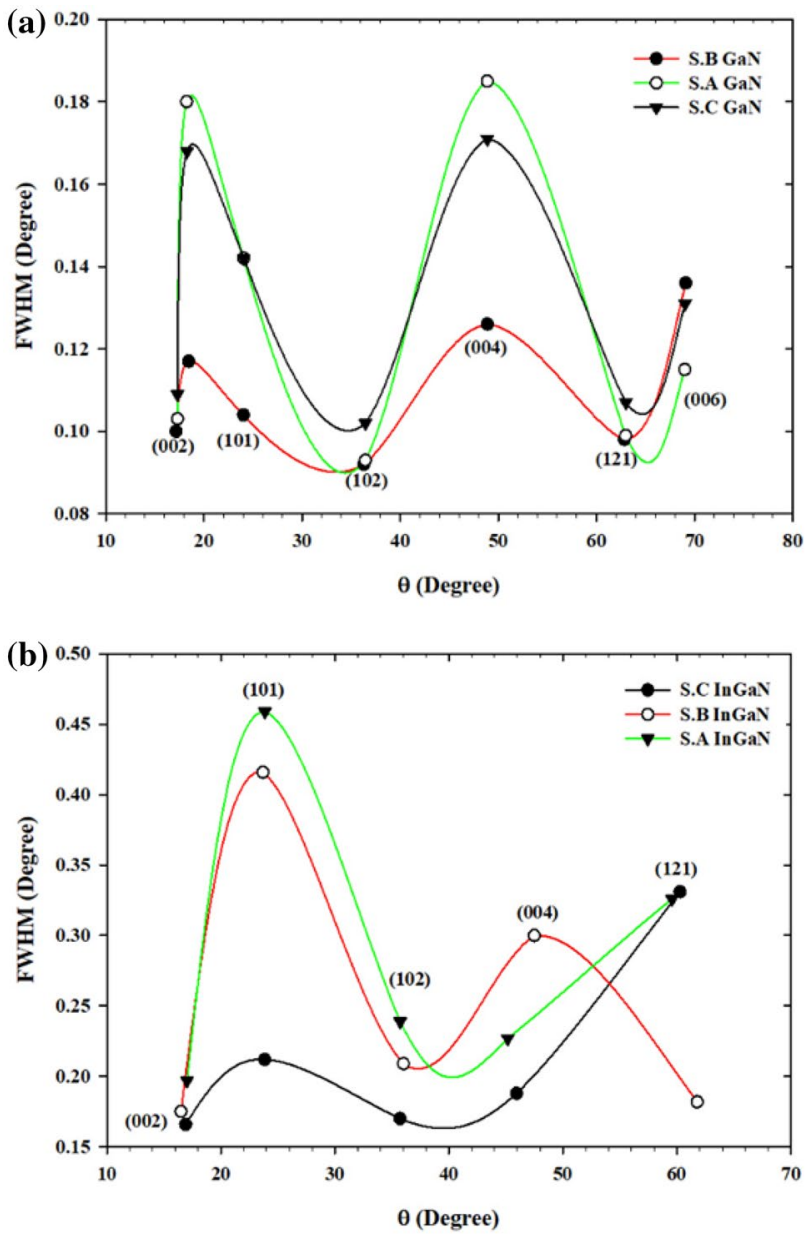

Fig. 4 a, b FWHM values dependent on (002), (101), (102), (004), (121), (006) Bragg diffraction angles for samples A, B and C GaN and InGaN layers

\subsubsection{Strain}

In-plane biaxial strain in epilayer can be calculated using Eq. (1). If biaxial stress along a direction is known [12, 13].

$\sigma_{f}=\left(c_{11}+c_{22}-2 \frac{c_{13}^{2}}{c_{33}}\right) \varepsilon_{a}^{b}$

Here strain $\left(\varepsilon_{a}^{b}\right)$ is calculated by division of difference between universal and experimental lattice parameters to experimental lattice parameter. Inside the parentheses in Eq. (2) is called axial module $\left(M_{f}\right)$. So biaxial strain is given as $\sigma_{f}=M_{f} \varepsilon_{a}^{b}$ [12].

Biaxial strains calculated for all samples are given in Fig. 5 dependent on temperature. Biaxial strain properties show increasing behaviour for $\mathrm{GaN}$ layer, but for InGaN layer it decrease with increasing temperature.

After $320^{\circ} \mathrm{C}$ for each layer biaxial strain value difference decrease with increasing temperature. The difference in the values of biaxial strain in a wide range of temperature is caused by the thermal expansion coefficient difference of $\mathrm{GaN}$ and InGaN or a wafer bending situation may be present. This wafer bending may be towards inside or outside the sample so irregular variation of biaxial strain-dependent on temperature is normal.

\subsubsection{Williamson-Hall (W-H)}

By scanning of symmetric reflections in radial direction, it is noticed that vertical crystal dimension perpendicular to bottom layer surface and heterogeneous strain along $c$ axis caused a broadening in the Bragg reflections. For symmetric and asymmetric scans, incident and reflected wave vectors are found in $z$-axis which contain reflection plane.

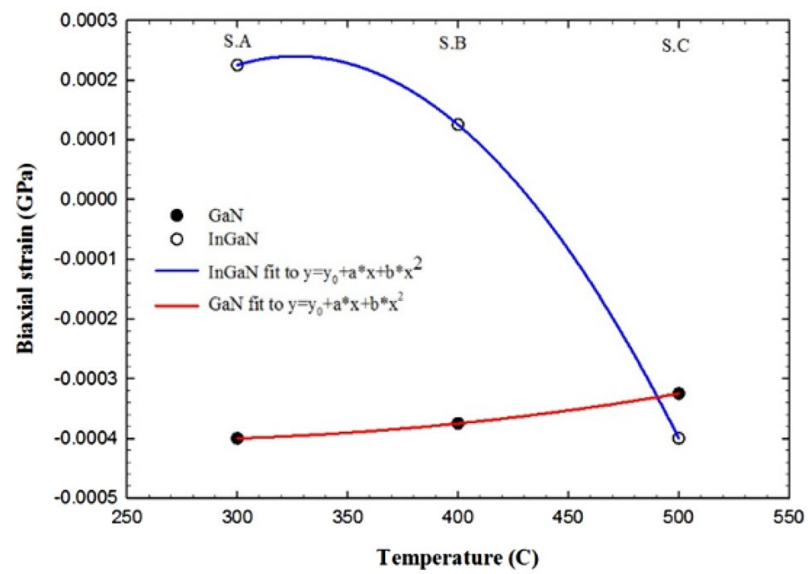

Fig. 5 Biaxial strain dependent on temperature for $\mathrm{GaN} / \mathrm{InGaN}$ structures 
Lateral crystal dimension and tilt of the mosaic blocks which are gained by symmetric reflections, cause a broadening in the peak plots of HR-XRD which are perpendicular to $z$ axis [14]. Contribution of these two effects causes a linear dependence on the broadening of reflection plane. This situation is used to separate the effect of each. For this aim, a Williamson-Hall plot can be used (W-H). This plot is a linear plot that we use FWHM as a function of reflection

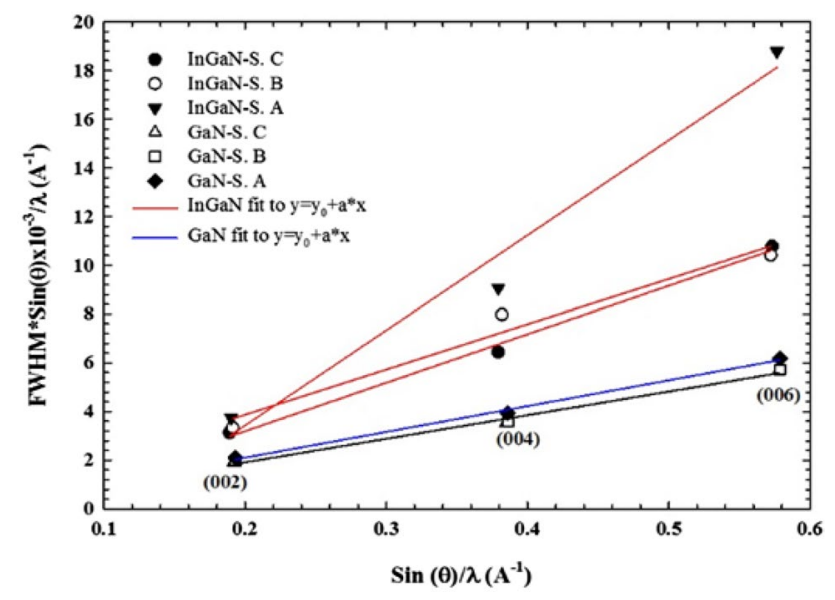

Fig. 6 Williamson-Hall plots for edge mosaic blocks in (002), (004), (006) planes for samples A, B and C InGaN and GaN layers degrees gained from rocking curves. This plot is formed by plotting $\mathrm{FWHM} \times \sin (\theta) / \lambda$ function versus $\sin (\theta) / \lambda[17,18]$.

In Fig. $6 \mathrm{WH}$ plots for $\mathrm{GaN}$ and InGaN layers in (002), (004), (006) planes are shown. FWHM is the total width of the measured profile, $\lambda$ and $\theta$ are wavelength and incident angle of X-rays, respectively. Tilt angle of plane is gained from the slope of $\mathrm{W}-\mathrm{H}$ plot and length of the lateral mosaic block $\left(L_{\|}\right)$is gained from the $y$-axis intercept of the fit. These fits are described by a line equation as $y=y_{0}+a \times x$. For S.A InGaN layer $y_{0}$ and a values are measured as (-4.37), (39), for S.B InGaN layer, (0.16), (18.55), for S.C InGaN layer, $(-0.78),(19.89)$, for S.A GaN layer, $(-0.0126),(10.59)$ and for S.B and C GaN layer, (0.026), (9.7), respectively. W-H plot has two limitations. Firstly, it is highly crystalized and the slope gets near to zero. Second limitation is, it may be far from a crystal which means having an amorphous structure or weakly dimensioned poly crystal structure. In this study, weak crystalized structure, lateral and vertical crystal lengths are determined between these two limits. Data gained from calculations can be seen in Table 1 .

For GaN layer, tilt angles for S.A are approximately the same at all temperature steps except for 400 and $450{ }^{\circ} \mathrm{C}$. There is a decrease at $400{ }^{\circ} \mathrm{C}$ and a sharp increase at $450{ }^{\circ} \mathrm{C}$. For S.B there is no considerable difference in tilt angle values with increasing temperature. The situation is the same for S.C. Lateral crystal length values for $\mathrm{GaN}$

Table 1 Tilt, lateral length, vertical length of $\mathrm{InGaN}$ and $\mathrm{GaN}$ layers

\begin{tabular}{|c|c|c|c|c|c|c|c|c|c|}
\hline \multirow[t]{2}{*}{ Temperature $\left({ }^{\circ} \mathrm{C}\right)$} & \multicolumn{3}{|l|}{ Sample A } & \multicolumn{3}{|l|}{ Sample B } & \multicolumn{3}{|l|}{ Sample C } \\
\hline & $\begin{array}{l}\text { Vertical (nm) } \\
\left(\times 10^{3}\right)\end{array}$ & $\begin{array}{l}\text { Lateral }(\mathrm{nm}) \\
\left(\times 10^{4}\right)\end{array}$ & Tilt & $\begin{array}{l}\text { Vertical (nm) } \\
\left(\times 10^{3}\right)\end{array}$ & $\begin{array}{l}\text { Lateral }(\mathrm{nm}) \\
\left(\times 10^{2}\right)\end{array}$ & Tilt & $\begin{array}{l}\text { Vertical (nm) } \\
\left(\times 10^{3}\right)\end{array}$ & $\begin{array}{l}\text { Lateral }(\mathrm{nm}) \\
\left(\times 10^{2}\right)\end{array}$ & Tilt \\
\hline \multicolumn{10}{|l|}{300} \\
\hline $\mathrm{GaN}$ & 1.7 & 9 & 321.4 & 1.7 & 9 & 321.4 & 1.8 & 22.5 & 300 \\
\hline InGaN1 & 4.2 & 9 & 264.7 & 5 & 9 & 450 & 6.8 & 5.63 & 187.5 \\
\hline InGaN2 & 4.2 & 9 & 264.7 & 5 & 9 & 450 & 6.8 & 5.63 & 187.5 \\
\hline \multicolumn{10}{|l|}{350} \\
\hline $\mathrm{GaN}$ & 1.7 & 22.5 & 321.4 & 1.6 & 1.5 & 321.4 & 1.8 & 22.5 & 300 \\
\hline InGaN1 & 3.8 & 22.5 & 204.5 & 4.3 & 9 & 225 & 6.8 & 5.63 & 187.5 \\
\hline InGaN2 & 3.8 & 2.2 & 204.5 & 4.3 & 9 & 225 & 6.8 & 5.63 & 187.5 \\
\hline \multicolumn{10}{|l|}{400} \\
\hline $\mathrm{GaN}$ & 1.1 & 0.1 & 321.4 & 1.7 & 5 & 321.4 & 1.8 & 22.5 & 300 \\
\hline InGaN1 & 5.4 & 5.6 & 450 & 3 & 6.4 & 150 & 6.8 & 5.63 & 187.5 \\
\hline InGaN2 & 5.4 & 5.6 & 450 & 3 & 6.4 & 150 & 6.8 & 5.63 & 187.5 \\
\hline \multicolumn{10}{|l|}{450} \\
\hline $\mathrm{GaN}$ & 1.9 & 0.6 & 375 & 1.7 & 5 & 346 & 1.8 & 22.5 & 300 \\
\hline InGaN1 & 2 & 5 & 150 & 3.1 & 75 & 166.6 & 6.8 & 5.63 & 187.5 \\
\hline InGaN2 & 2 & 5 & 150 & 3.1 & 75 & 166.6 & 6.8 & 5.63 & 187.5 \\
\hline \multicolumn{10}{|l|}{500} \\
\hline $\mathrm{GaN}$ & 1.7 & 2.2 & 346 & 1.8 & 1.5 & 346 & 1.8 & 22.5 & 300 \\
\hline InGaN1 & 4.7 & 11.3 & 195 & 3.1 & 225 & 180 & 6.8 & 5.63 & 188 \\
\hline InGaN2 & 4.7 & 11.3 & 195 & 3.1 & 225 & 180 & 6.8 & 5.63 & 188 \\
\hline
\end{tabular}


layer shows fluctuations with increasing temperature. At $350{ }^{\circ} \mathrm{C}$ it sharply increase and at $400{ }^{\circ} \mathrm{C}$ it again sharply decrease. At 450 and $500{ }^{\circ} \mathrm{C}$ it slightly increase for S.A. For S.B. lateral crystal length values vary softer than S.A. For S.C. the same values do not change with increasing temperature. Vertical crystal length values for all three samples do not show a big difference with increasing temperature. For InGaN1 layer, tilt angle values for S.A. slightly fluctuate with increasing temperature. For S.B. the situation is the same as in S.A. For S.C. tilt angle values do not change with an increase in temperature. Lateral crystal length values, sharply increase at $350{ }^{\circ} \mathrm{C}$ and suddenly decrease at $400{ }^{\circ} \mathrm{C}$, after this temperature value it softly increase. For S.B. lateral crystal length values shows a sudden increase at 450 and $500{ }^{\circ} \mathrm{C}$. For S.C. the same values for lateral crystal length can be seen at all temperatures. Vertical crystal length values for S.A. makes a peak at $400{ }^{\circ} \mathrm{C}$, at other temperatures they do not show considerable variation. For S.B. vertical crystal length values do not change too much with increasing temperature, but at $300{ }^{\circ} \mathrm{C}$ its value is the highest. For S.C. vertical crystal length values does not change with increasing temperature. For InGaN2 layer, tilt angle values show a fluctuative behaviour for S.A and B with increasing temperature but, for S.C these values are constant. The situation is the same for lateral crystal length values. Vertical crystal length values for S.A makes a peak at $400{ }^{\circ} \mathrm{C}$, the values does not change for other temperatures. For S.B vertical crystal length values are approximately the same at all temperature steps except for $300^{\circ} \mathrm{C}$. And for S.C values of vertical crystal length are the same for all temperature steps. In addition to this, mosaic block lengths which are parallel to surface for GaN layers are higher than the ones for InGaN. This situation may be caused by rapid cooling of the samples. As a result of this, layers are formed as mosaic blocks. Also in plotting $\mathrm{W}-\mathrm{H}, \mathrm{FWHM} \times \cos (\theta) / \lambda$ reflection versus $\sin (\theta) / \lambda$ gives a straight line. $Y$-axis intercept of this plot gives perpendicular mosaic block length $\left(y_{0}\right)$. Using $L_{\perp}=0.9 /\left(2 y_{0}\right)$ equation we can calculate $L_{\perp}$. Stress value $\varepsilon_{\perp}$ is calculated by the slope of the fit as $4 \varepsilon_{\perp}$ directly $[14,15]$.

In Fig. 7, W-H plots can be seen for InGaN and GaN layers. These linear plots are described by a line equation as $y=y_{0}+a \times x$. For S.A InGaN layer $\mathrm{y}_{0}$ and a values are measured as (14), (-6.4), for S.B InGaN layer (14), (-13), for S.C InGaN layer (12), (-11), for S.A GaN layer, (8.6), (-9.3), for S.B GaN layer (8.1), (-8.9), for S.C GaN layer, (7.9), (-8.5), respectively. In Table 1 perpendicular crystal length values for $\mathrm{GaN}$ can be seen. These values decrease and increase with increasing temperature softly. The same values for InGaN continuously decrease except at $400{ }^{\circ} \mathrm{C}$.

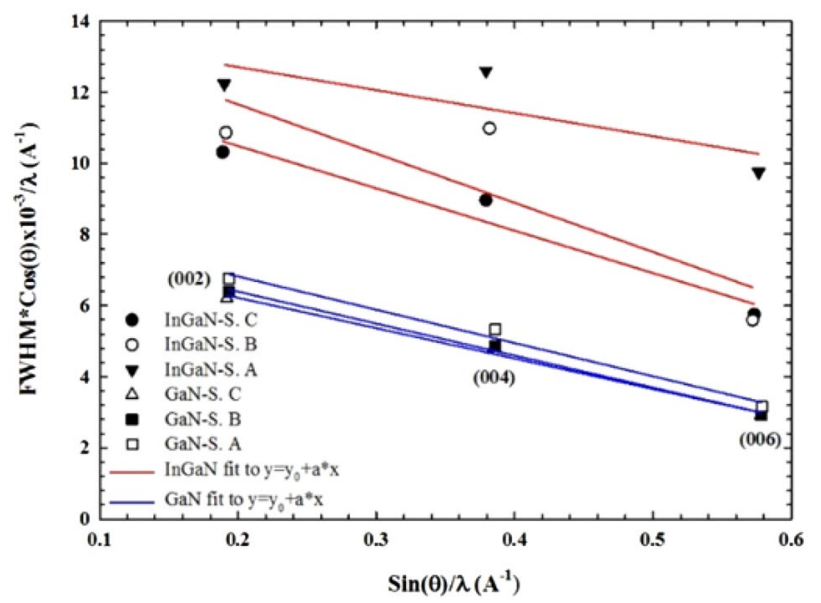

Fig. 7 Williamson-Hall plots for vertical mosaic blocks in (002), (004) and (006) planes for samples A, B and C InGaN and GaN layers

\subsubsection{Edge and screw dislocations}

Dislocations between $10^{8}$ and $10^{12} \mathrm{~cm}^{-2}$ are accepted as threadening dislocations (TD) and nitride-based layers deposited on sapphire, silicon carbide, and silicon wafers which has lattice mismatch show threadening dislocation behaviour $[15,16]$.

In this study, $\mathrm{GaN}$ and $\mathrm{InGaN}$ layers are deposited on sapphire which show high-threadening dislocation density. Dislocations on such layers have three types [20,23]. These are edge dislocation given by Burgers vector $<a>$, screw dislocation given by Burgers vector $\langle\mathrm{c}\rangle$ and mixed type dislocation given by Burgers vector $\langle\mathrm{c}+\mathrm{a}\rangle$. To see the results caused by crystallisation level and mosaic defects, two methods are used. In the first method, edge $\left(D_{\text {edge }}\right)$ and screw $\left(\mathrm{D}_{\text {screw }}\right)$ type dislocations in epitaxial layers is calculated by the following equation [17-21];

$D_{\text {screw }}=\frac{\beta_{(0002)}^{2}}{9 b_{\text {screw }}^{2}}, D_{\text {edge }}=\frac{\beta_{(10-12)}^{2}}{9 b_{\text {edge }}^{2}}$

Here $\beta$ is the FWHM value and it is measured by HRXRD rocking curves. It determines the crystallite state (for $\mathrm{GaN} \mathrm{b}_{\text {screw }}=0.5185 \mathrm{~nm}, \mathrm{~b}_{\text {edge }}=0.3189 \mathrm{~nm}$ and for InGaN $\mathrm{b}_{\text {screw }}=0.5198 \mathrm{~nm}, \mathrm{~b}_{\text {edge }}=0.3417 \mathrm{~nm}$.) According to second method, edge and screw dislocation densities are dependent on Burgers vector, tilt and lateral mosaic length. All kind of dislocations are dependent on lateral mosaic length, tilt and twist angles. As reported by Metzger et al. [12], GaN films in (002) average twist angle is related with edge Burgers vector $[b=1 / 3(11-20)]$ type dislocation density. Also average tilt angle, is related with monotonous Burgers vector $b=(0001)$ screw type dislocation [11]. Burgers vector is formed by 
azimuthal turning of crystal block around the normal of $[b=1 / 3(11-20)]$ edge type dislocation surface. Measured twist is gained from twist angle $\alpha_{\text {twist }}$. Using these information we can calculate edge type dislocation. If dislocations are gathered on the border of small particle size, edge type dislocation densities are calculated with the following equations [22, 23]

$N_{\text {screw }}=\frac{a_{\text {tilt }}^{2}}{4.35 b_{\text {screw }}^{2}}, N_{\text {edge }}=\frac{a_{\phi}}{2.1 b_{\text {edge }} L \|}$

Here $\alpha_{\text {tilt }}$ angle is a mosaic defect tilt angle. $\alpha_{\phi}$ is the peak broadening of asymmetric planes and $b_{\text {screw }}$, is the length of Burgers vector.

Edge and screw type TD densities calculated for $\mathrm{GaN}$ and InGaN layers using second method are given in Table 2.

According to second method, edge type dislocations first decrease, later increase and screw type dislocations first increase, later decrease with an increase in temperature for $\mathrm{GaN}$ and InGaN layers. The situation is the same for all three samples. If Table 2 is examined carefully, one can see that TD densities are lower for GaN layers in general. This result can be attributed to growth conditions. Growth conditions are more optimised for $\mathrm{GaN}$ layers if compared with InGaN layers.

\subsection{Optical characteristics}

\subsubsection{PL}

Photoluminescence (PL) measurements were made by using the Jobin Yvon Fluorolog-550 PL system with a $50 \mathrm{~mW}$ $\mathrm{HeCd}$ laser $(\lambda=325 \mathrm{~nm})$ as excitation light source at room temperature.

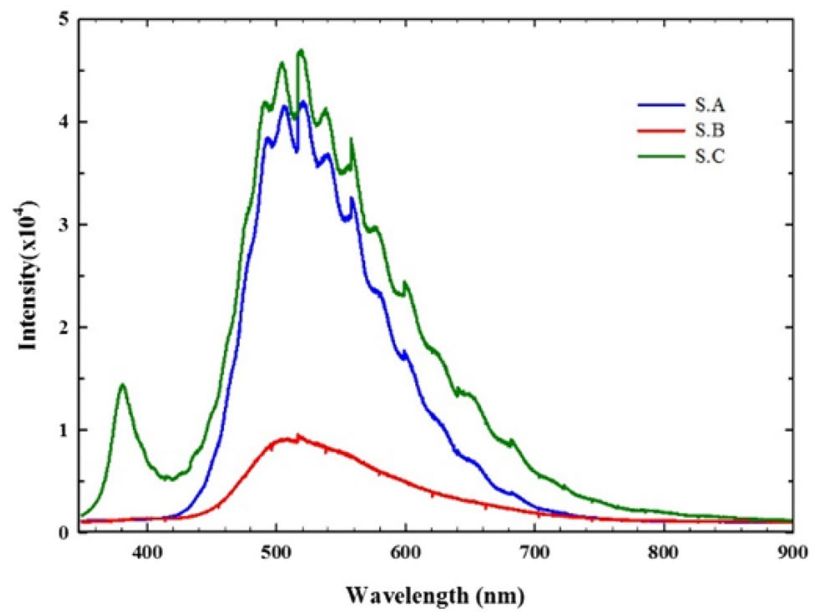

Fig. 8 PL spectra of InGaN/GaN structure at room temperature
Table 2 Screw and edge dislocation densities of $\mathrm{GaN}$ and $\mathrm{InGaN}$ layers dependent on temperature

\begin{tabular}{|c|c|c|c|c|c|c|}
\hline \multirow[t]{2}{*}{ Temperature $\left({ }^{\circ} \mathrm{C}\right)$} & \multicolumn{2}{|l|}{ Sample A } & \multicolumn{2}{|l|}{ Sample B } & \multicolumn{2}{|l|}{ Sample C } \\
\hline & Edge D.L $\left(\times 10^{9}\right)$ & $\begin{array}{l}\text { Screw } \\
\text { D.L } \\
\left(\times 10^{8}\right)\end{array}$ & Edge D.L $\left(\times 10^{9}\right)$ & $\begin{array}{l}\text { Screw } \\
\text { D.L } \\
\left(\times 10^{8}\right)\end{array}$ & $\begin{array}{l}\text { Edge D.L } \\
\left(\times 10^{9}\right)\end{array}$ & $\begin{array}{l}\text { Screw } \\
\text { D.L } \\
\left(\times 10^{8}\right)\end{array}$ \\
\hline \multicolumn{7}{|l|}{300} \\
\hline $\mathrm{GaN}$ & 1.55 & 1.25 & 2.36 & 1.25 & 1.55 & 1.25 \\
\hline InGaN1 & 9.55 & 1.64 & 10.80 & 9.55 & 9.55 & 1.64 \\
\hline InGaN2 & 10.80 & 1.60 & 42.90 & 3.62 & 4.22 & 3.57 \\
\hline \multicolumn{7}{|l|}{350} \\
\hline $\mathrm{GaN}$ & 1.46 & 1.25 & 2.22 & 1.29 & 1.46 & 1.25 \\
\hline InGaN1 & 1.50 & 3.39 & 4.80 & 3.56 & 1.50 & 3.39 \\
\hline InGaN2 & 1.60 & 3.20 & 4.00 & 3.01 & 0.16 & 3.20 \\
\hline \multicolumn{7}{|l|}{400} \\
\hline $\mathrm{GaN}$ & 1.42 & 3.10 & 1.69 & 1.26 & 1.40 & 3.00 \\
\hline InGaN1 & 2.21 & 1.30 & 2.90 & 4.59 & 2.21 & 1.30 \\
\hline InGaN2 & 1.67 & 4.89 & 1.89 & 4.79 & 1.28 & 3.86 \\
\hline \multicolumn{7}{|l|}{450} \\
\hline $\mathrm{GaN}$ & 1.46 & 9.70 & 3.28 & 1.16 & 0.34 & 1.49 \\
\hline InGaN1 & 3.13 & 4.13 & 3.14 & 4.31 & 3.10 & 4.10 \\
\hline InGaN2 & 3.14 & 4.31 & 2.86 & 1.31 & 0.34 & 1.47 \\
\hline \multicolumn{7}{|l|}{500} \\
\hline $\mathrm{GaN}$ & 1.50 & 1.08 & 2.24 & 1.19 & 0.35 & 1.49 \\
\hline InGaN1 & 1.73 & 3.81 & 1.90 & 3.82 & 1.67 & 4.89 \\
\hline InGaN2 & 1.90 & 3.82 & 2.86 & 1.31 & 0.34 & 1.47 \\
\hline
\end{tabular}


Figure 8 shows, carrier density dependence of PL spectra gained by the excitation caused by this laser. Vertical axis is formed on a logarithmic scale to investigate spectral variation in a wide range. About $380(3.24 \mathrm{eV}) \mathrm{nm}$ wavelength, one of the components of $\mathrm{GaN}$ for S.C caused a sharp peak [24]. This shows that carrier density belonging to this component is high in this spectral region. Each three component gave a broad green emission centered at about $\sim 520 \mathrm{~nm}(2.38 \mathrm{eV})$ wavelength. The origin of the this emission is attributed to several impurities and native defects [25]. The wavelength coincide with this maxima can be used to calculate the band gap $(\mathrm{Eg})$ using $E=h \times c / \lambda$ equation [26]. S.A, S.B and S.C., respectively, the band gap of $2.4 \mathrm{eV}$, $2.35 \mathrm{eV}$ and $2.38 \mathrm{eV}$. After $650 \mathrm{~nm}$ wavelength, the decrease in PL intensity axis means after this value any component of InGaN has an energy level. For stimulation which results in excitation, the energy of the incident photon must be absolutely equal to one of the energy levels of the atom [28].

\subsubsection{Transmission}

Transmission can be defined as the ratio of intensity of transmitted light to the intensity of the incident light $\left(T=I / I_{0}\right)$. Transmission and reflection are both dependent on the refraction and absorbtion properties of the material. Optical transmission measurements are used for determining the optical absorption coefficients and some impurities. Impurities coincide with the optical measurements. Some impurities have characteristic absorbtion lines because of vibrational modes. For example Ga and $\mathrm{N}$ in InGaN. A photon absorbed in a semiconductor produce local vibrational modes around some impurities and cause some vibrations [27]. During transmission measurements, photons come to the sample and transmission is measured as a function of wavelength. The band gap of the semiconductor can be determined by the measurement of the absorbtion coefficient as a function of photon energy. Photons having greater energy than the band gap is absorbed [28].

In this study, the optical transmittance measurements were performed by UV-Vis spectrometer (Lambda $2 \mathrm{~S}$, Perkin Elmer) at room temperature in the range of $200-1100 \mathrm{~nm}$. Spectra of transmission measurements is gained by plotting wavelength versus $\% \mathrm{~T}$. This plot is shown in Fig. 9 According to this plot, light is completely absorbed in the near visible region $(\sim 390 \mathrm{~nm})$. The red curve estimated as belonging to S.A increased more sharply than S.B and S.C curves. The reason for this may be, the band gap of $\mathrm{GaN}$ is in the blue region. On the other sides of the spectra, there is no great difference except the fluctuations of the amplitude of the intensity. The difference for the amplitude of the fluctuations may be caused by the difference in the thicknesses. Thicknesses may cause the layers to act as semitransparent windows [28]. Interference fringes was formed

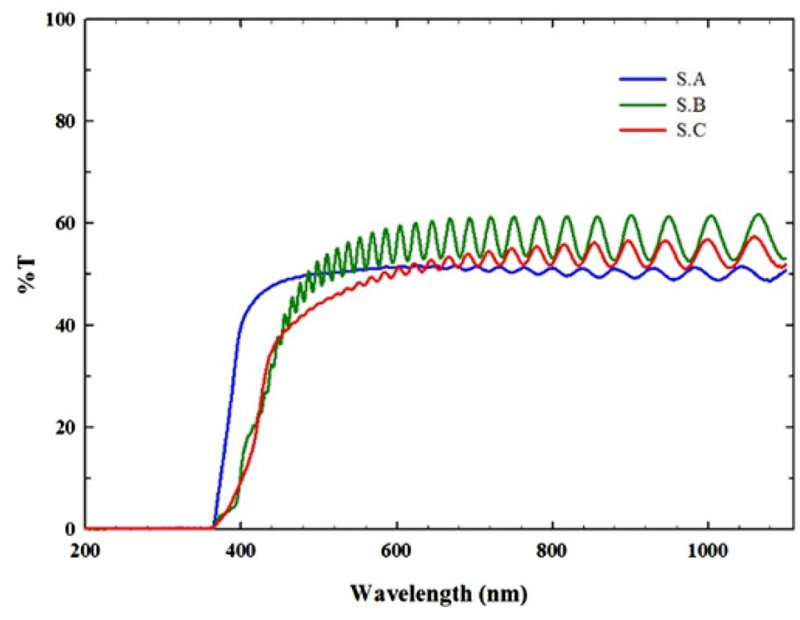

Fig. 9 Transmission spectra of InGaN/GaN structure at room temperature

in transmittance spectra dependent on the change of film thickness. The bandgaps are estimated by the high-energy edges of the interference fringes [29]. But this absorption edge could be due to excitons rather than band-to-band transitions which correspond to the energy gap. There is no unique procedure which allows the determination of an energy gap from the measured transmission spectrum. Because of that, PL measurement was used for determination of bandgap. No light absorbtion is seen in transmission spectra in Fig. 9 at approximately $520 \mathrm{~nm}$. But in PL spectra there is a peak near to $520 \mathrm{~nm}$ wavelength. The reason for this may be that $520 \mathrm{~nm}$ is the wavelength of green light, but InGaN band gap is in blue region. So InGaN active layers in samples could not detect $520 \mathrm{~nm}$ wavelength green light. Additionally, in graded samples there may occur a shift in the band gap of the structures forming the alloy.

\subsubsection{FTIR}

Fourier transform spectroscopy is successful in gaining spectra when the low light energy is a problem. In this study FTIR optical spectrums of InGaN and GaN were recorded on a Bruker Vertex 80 IR spectrometer in the range of $7500-400 \mathrm{~cm}^{-1}$. FTIR transmission measurements performed in normal incidence also formed interference fringes in FTIR transmittance spectra. In addition, the transmission measurements at $4000-400 \mathrm{~cm}^{-1}$ are performed with ATR (attenuated total reflector) unit. Because the ATR method uses evanescent waves (EWs), which can directly excite the Surface phonon polariton and this provides the direct observation that LO and TO (longitudinal and transverse) optic phonon modes [30]. The ATR spectrum of all samples show a sharp absorption peak at the range of $670-690 \mathrm{~cm}^{-1}$. The observed peak is a result of the resonance at a frequency 
where the wave vectors of the incident radiation and the surface interface polaritons are matched [31].

The FTIR spectra of InGaN solar cell can be seen in Fig. 10. This spectra shows the plot of wavenumber versus transmittance. The common three features for each sample may be seen in the spectra.

1. In $2000-1000 \mathrm{~cm}^{-1}$ spectral range, intensity of In related strained bands during growth decrease. This is caused by In- $\mathrm{Ga}$ and $\mathrm{Ga}-\mathrm{N}$ bonds [32].

2. In $3000-3500 \mathrm{~cm}^{-1}$ spectral range the increase in the amplitude of the fluctuations and transmittance can be seen. This is related with InGaN bonds. These are the LO and TO phonons around this spectral range [32].

3. On the other sides of the spectra there can be seen ordinary fluctuations and there are no sharp bands [32].

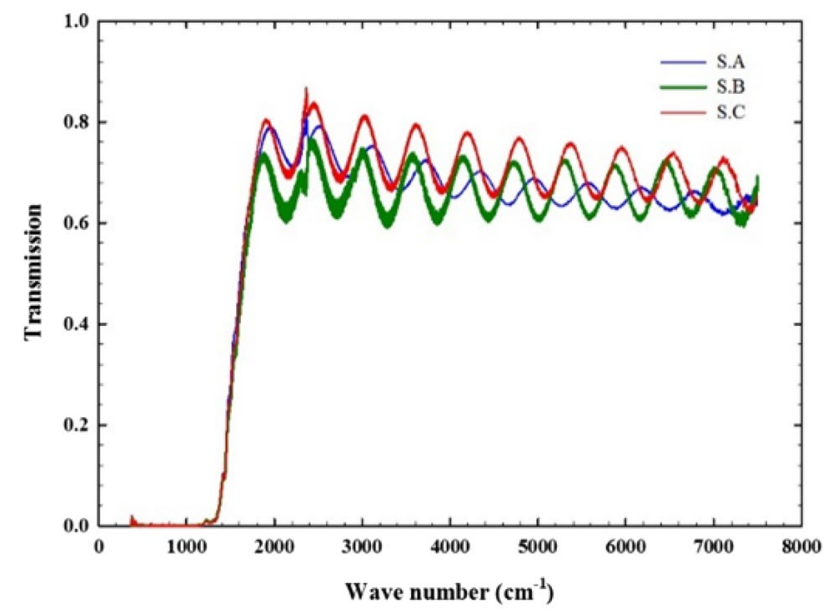

Fig. 10 FTIR vibration spectra of InGaN active layer
All the properties in the spectra, can be compared by making more than one measurement. Photometric accuracy can be compared by the thickness of the curves in spectra [33]. Taking into account the intensity, the maxima about $2000 \mathrm{~cm}^{-1}$ is caused by vibrational modes of transmitted electrons. Also this maxima may be caused by the structural defects which contain In. The aim of choosing FTIR measurement to analyze LO and TO phonons is to show that they can also be detected by using FTIR. The best way to analyze them is Raman scattering. That is made in another study of the authors. FTIR measurement uses low energy photons. Raman uses Laser wavelength of 500-800 $\mathrm{nm}$ range. The intensity of LO and TO phonons gained from FTIR are less than the ones from Raman, but they can still be detected as shown in Fig. 10.

As can be seen in Fig. 2, the XRD pattern of these three samples, S.A has the best crystal quality. Because the peaks of InGaN and GaN are fully distorted from each other. In optical spectra for PL it can be seen that S.A has the most narrow peak, this result is in good accordance with XRD pattern. In Transmission spectra S.A plot has the best and sudden tilt. So among these three samples S.A is best because of the optimised growth conditions. These structures may be used as blue light-emitting diodes or solar cells.

\subsubsection{AFM}

Morphological properties of the samples are characterised by high performance Atomic Force Microscope using the dynamic scanning mode.

Figure 11 shows AFM images of sample A, B, C with $5 \times 5 \mu \mathrm{m}^{2}$ scanning area (3D). Surface of S.A is more homogeneous with respect to S.B and C. Sample A has less smooth surface morphology. Root mean square (RMS) values which are used in determining the surface roughness (a)

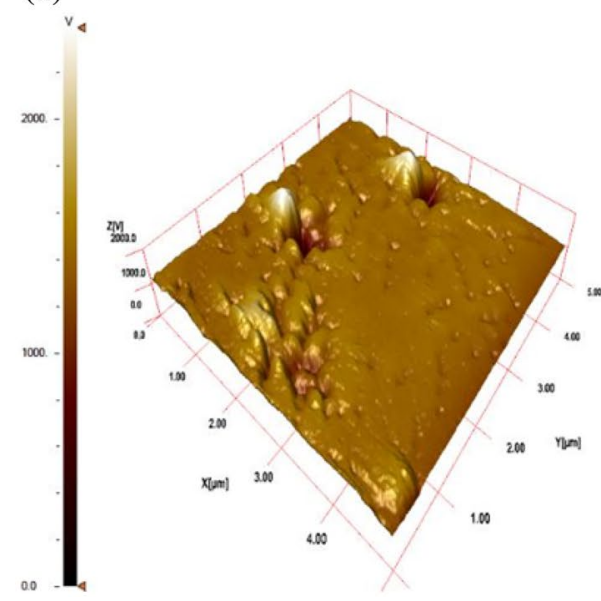

(b)

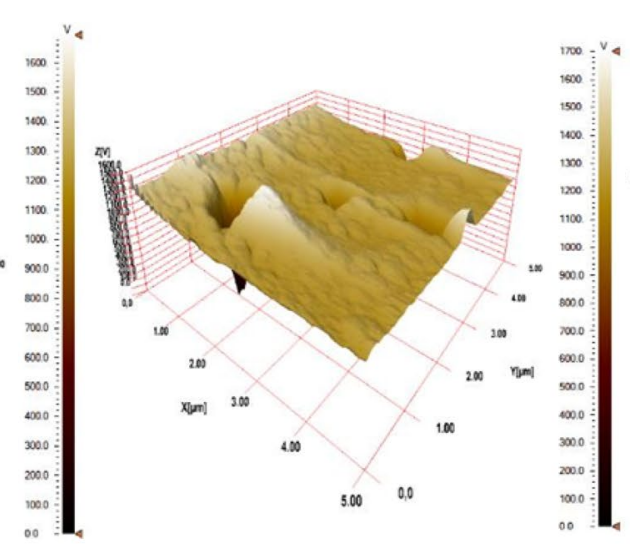

(c)

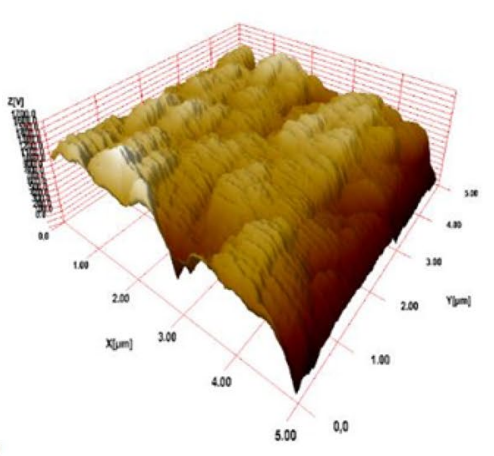

Fig. $113 \mathrm{D} 5 \times 5 \mu \mathrm{m}^{2}$ scanning area AFM images of InGaN/GaN structures. a S.A, b S.B, c S.C at room temperature 
of the samples are $4.883 \mathrm{~nm}, 10.225 \mathrm{~nm}$ and $10.045 \mathrm{~nm}$ for S.A, B and C respectively. Variation of RMS values are related with increasing of particle size. These results are in accordance with particle size calculations by $\mathrm{W}-\mathrm{H}$ method. Surface of S.A and B layer includes more packed and white regions. These white regions are the oxide or white rust formed on the surface of the samples by touching the air. In S.A there are less white regions and in this layer dark colour region is more dense. In image of S.A, white and pyramid shaped areas can be seen. The pyramid shaped hills may be Ga and white colour may be the oxide on it. On the surface of all three samples, black dots and randomly directed hills can be seen. This shows that all the samples are grown in steps. Because growth with steps shows smooth surface morphology it effects device performance in a good way [34]. On the surface of S.A, B, C it is seen that randomly directed hills are combined with black dots. On the surface of GaN layers grown in steps, dots combined to these hills are related with screw type dislocations [35]. Surface roughness is closely related with hill widths on GaN surfaces. As hill width increase surface roughness decrease. Surface images of samples are similar with those in literature. AFM images are a good indicator of surface morphology of InGaN and $\mathrm{GaN}$ structures. These images show that variations during growth, effects surface morphology.

\section{Conclusion}

Mosaic parameters of InGaN structures such as lateral and vertical mosaic length, edge and screw dislocations were determined by HR-XRD technique. For GaN and InGaN layers, AFM, FTIR, PL and transmission properties were investigated. It is seen that results are in accordance with those in literature. AFM results showed that particle size is compatible with those gained from $\mathrm{W}-\mathrm{H}$ method. FTIR, $\mathrm{PL}$ and transmission measurements indicate that band gap is approximately the same with the value found in literature and previous works. Pyramid-like shapes in AFM images with white colour showed that there formed an oxide layer or white rust over Ga on the surface of samples. PL spectra showed that band gap shifted towards blue region in the electromagnetic spectrum. All these results indicate that, structural, morphological and optical properties are in agreement with each other with increasing temperature. According to XRD pattern in Fig. 1 and optical spectra it is seen that InGaN layer in S.A is more optimised than others.

\section{References}

1. H. Morkoc, Hand book of nitride semiconductors and devices, vol. 1 (Wiley, Berlin, 2008), p. 16
2. S. Çörekçi (2008). Morphological characteristics of III-V group compound semiconductors Doctora Thesis, Gazi University, Ankara, 10(11), p. 39

3. S.T. Bayrak (2011). InGaN/GaN Multi quantum well light emitting diodes. Doctora thesis Balıkesir University Science institude, Balıkesir, p. 7-11

4. S. Nakamura, The roles of structural imperfections in InGaNbased blue light-emitting diodes and laser diodes. Science 281(5379), 956-961 (1998)

5. S. Nakamura, I. Akasaki, H. Amano, Blue, LEDs, Filling the world with new light (The royal Swedish academy of sciences, Stockholm)

6. D.M. Graham, A. Soltani-Vala, P. Dawson, M.J. Godfrey, T.M Smeeton, J.S. Barnard, M.J. Kappers, C.J. Humphreys, E.J. Thrush, J. Appl. Phys. 97, 103508 (2005)

7. F.A. Ponce, B.S. Krusor, J.S.M. Jr, W.E. Plano, J. Welch, Microstructure of GaN epitaxy on SiC using AlN buffer layers. Appl. Phys. Lett. 67(3), 410-412 (1995)

8. S. Chichibu, T. Azuhata, T. Sota, S. Nakamura, Spontaneous emission of localized excitons in InGaN single and multiquantum well structures. Appl. Phys. Lett. 69(27), 4188-4190 (1996)

9. S.D. Lester, F.A. Ponce, M.G. Crawford, D.A. Steigerwald, High dislocation densities in high-efficiency Gan-based light-emittingdiodes. Appl. Phys. Lett. 66(10), 1249-1251 (1995)

10. M.K. Öztürk, H. Yu, B. Sarıkavak, S. Korçak, S. Özçelik, E. Özbay, Structural analysis of an InGaN/GaN based light emitting diode by X-ray diffraction. J. Mater. Sci. 21(2), 185-191 (2010)

11. M.K. Öztürk, S. Çörekçi, M. Tamer, S. Çetin, S. Özçelik, E. Özbay, Microstructural properties of InGaN/GaN light-emitting diode structures with different In content grown by MOCVD. Appl. Phys. A Mater. Sci. Process. 114(4), 1215-1221 (2014)

12. V.S. Harutyunyan, A.P. Aivazyan, E.R. Weber, Y. Kim, Y. Park, S.G. Subramanya, High resolution X-ray diffraction strain-stress analysis of GaN/Sapphire heterostructures. J. Phys. D Appl. Phys. 34(10A), A35-A39 (2001)

13. M.A.G. Halliwell, X-ray diffraction solutions to heteroepitaxial growth problems. J. Cryst. Growth 170(1-4), 47-54 (1997)

14. T. Metzger, R. Höpler, E. Born, O. Ambacher, M. Stutzmann, R. Stömmer, M. Schuster, H. Gobe, S. Christiansen, M. Albrecht, H.P. Strun, Defect structure of epitaxial GaN films determined by transmission electron microscopy and triple-axis X-ray diffractometry. Philos. Mag A 77(4), 1013-1025 (1998)

15. M.K. Öztürk, H. Altuntaş, S. Çörekçi, Y. Hongbo, S. Özçelik, E. Özbay, Strain-stress analysis of AlGaN/GaN heterostructures with and without an AlN suffer and interlayer. Strain 47(s2), 19-27 (2011)

16. M.K. Öztürk, E. Arslan, İ Kars, S. Özçelik, E. Özbay, Strain analysis of the $\mathrm{GaN}$ epitaxial layers grown on nitridated $\mathrm{Si}(111)$ substrate by metal organic chemical vapor deposition. Mater. Sci. Semiconduct. Process. 16(1), 83-88 (2013)

17. G.K. Williamson, W.H. Hall, X-ray line broadening from filed aluminium and wolfram. Acta Metall. 1(1), 22-31 (1953)

18. E. Arslan, M.K. Öztürk, Ö Duygulu, A.A. Kaya, S. Özçelik, E. Özbay, The influence of nitridation time on the structural properties of GaN grown on Si(111) substrate. Appl. Phys. A Mater. Sci. Process. 94(1), 73-82 (2009)

19. E. Arslan, M.K. Öztürk, A. Teke, S. Özçelik, E. Özbay, Buffer optimization for crack-free $\mathrm{GaN}$ epitaxial layers grown on $\mathrm{Si}(111)$ substrate by MOCVD. Jpn. J. Phys. D 41(15), 155317-155326 (2008)

20. M.E. Vickers, M.J. Kappers, R. Datta, C. McAleese, T.M. Smeeton, F.D.G. Rayment, C.J. Humphreys, In-plane imperfections in GaN studied by X-ray diffraction. J. Phys. D 38(A10), A99-A104 (2005)

21. X.H. Zheng, H. Chen, Z.B. Yan, Y.J. Han, H.B. Yu, D.S. Li, Q. Huang, J.M. Zhou, Determination of twist angle of in-plane 
mosaic spread of GaN films by high-resolution X-ray diffraction. J. Cryst. Growth 255(1-2), 63-67 (2003)

22. P. Gay, P.B. Hirsch, A. Kelly, The estimation of dislocation densities in metals from X-ray data. Acta Metall. 1(3), 315-319 (1953)

23. M.J. Hordon, B.L. Averbach, X-ray measurements of dislocation density in deformed copper and aluminum single crystals. Acta Metall. 9(3), 237-246 (1961)

24. A. Vogt et al., Recombination dynamics in planar and threedimensional $\mathrm{InGaN} / \mathrm{GaN}$ light emitting diode structures. J. Mater. Res. 32(13), 2456-2463 (2017)

25. M.A. Reshchikov, H. Morkoc, Luminescence properties of defects in GaN. J. Appl. Phys. 97(6), 135 (2005)

26. J.R. Taylor, C.D. Zafiratos, M.A. Dubson (2004). Modern Physics for scientists and engineers (Colorado University, Boulder), p. $101-115$

27. H. Çakmak (2012). Growth and characterisation of In rich InGaN solar cell epitaxial structures by metal organic chemical vapor deposition. Master Thesis, Middle East technical university, Turkey, 60-70

28. Y. Baş (2014). Investigation of InGaN blue LED microstructure defects from reciprocal space mapping. Doctora Thesis, Gazi University, Turkey, 60-103
29. J.F. Muth et al., Absorption coefficient, energy gap, exciton binding energy, and recombination lifetime of $\mathrm{GaN}$ obtained from transmission measurements. Appl. Phys. Lett. 71(18), 2572-2574 (1997)

30. S.S. $\mathrm{Ng}$ et al. Surface phonon polariton of wurtzite GaN thin film grown on c-plane sapphire substrate. Solid State Commun. 145(11-12), 535-538 (2008)

31. S.S. Ng et al. Surface and interface phonon polaritons of wurtzite $\mathrm{GaN}$ thin film grown on $6 \mathrm{H}-\mathrm{SiC}$ substrate. Appl. Phys. Lett. 94(24), 241912 (2009)

32. A. Erol Dilute III-IV nitride semiconductors and material systems. Phys. Technol. 2008, 105 (2008)

33. R. Darshan Sharma Jha, Analysis of structural, optical and magnetic properties of $\mathrm{Fe} / \mathrm{Co}$ co-doped $\mathrm{ZnO}$ nanocrystals. Ceram. Int. 43, 8488-8496 (2017)

34. A. Torabi et al., Surface and interface characterization of GaN/ $\mathrm{AlGaN}$ high electron mobility transistor structures by X-ray and atomic force microscopy. J. Vac. Sci. Technol. B 20(3), 1234 1237 (2002)

35. F.C. Frank, The influence of dislocations on crystal growth. Discuss. Faraday Soc. 5, 4854 (1949) 